\title{
To address emerging infections, we must invest in enduring systems: The kinetics and dynamics of health systems strengthening
}

\author{
Authors:* \\ Sonak Pastakia, PharmD, MPH, BCPS \\ Associate Professor of Pharmacy Practice \\ Purdue University \\ Benson Njuguna, BPharm \\ Clinical Pharmacist \\ Moi Teaching and Referral Hospital \\ Phuoc V. Le, MD, MPH \\ Assistant Professor, Internal Medicine and Pediatrics \\ University of California, San Francisco \\ Manisha K. Singh, BA \\ Project Coordinator \\ University of California, San Francisco \\ Tina Penick Brock, MSPH, EdD \\ Professor of Clinical Pharmacy \\ University of California, San Francisco \\ * All authors contributed to the writing of this manuscript and we acknowledge Conan \\ MacDougall, PharmD and Lydia Fischer, BA (MD candidate) for their contributions to \\ the work.

\section{Corresponding author:} \\ Tina Brock \\ UCSF Department of Clinical Pharmacy \\ 533 Parnassus Ave, Room U585 - Box 0622 \\ San Francisco, CA 94143 \\ Phone: (415) 476-5688 \\ Fax: (415) 476-6632 \\ Email: tina.brock@ucsf.edu
}

References: 5

Figures: $\quad 2$

Tables: $\quad 0$

Key words: capacity building, emerging infectious diseases, health systems plan, workforce

This is the author's manuscript of the article published in final edited form as:

Pastakia, S., Njuguna, B., Le, P. V., Singh, M. K., \& Brock, T. P. (2015). To address emerging infections, we must invest in enduring systems: The kinetics and dynamics of health systems strengthening. Clinical Pharmacology \& Therapeutics, 98(4), 362-364. http://dx.doi.org/10.1002/cpt.182 


\section{To address emerging infections, we must invest in enduring systems: The kinetics and dynamics of health systems strengthening}

Clinical pharmacology uses foundational principles of pharmacokinetics (PK) and pharmacodynamics (PD) to address medication use spanning a continuum from molecules to the masses. In the realm of infectious diseases, PK/PD attributes are considered especially important, because sub therapeutic dosing of antibiotics has been associated with poorer clinical outcomes in patients and increased incidences of drug resistance in populations. In consideration of these PK/PD principles, we will describe the analogous relationship between health systems strengthening, including for educating healthcare providers about emerging infections, and the tenets of therapeutic drug monitoring.

Worldwide, the populations bearing the greatest burden of emerging infectious diseases live in low- and middle-income countries (LMICs), where the typical tools for addressing these - such as preparedness plans, stockpiles of drugs and vaccines, and the health professionals to initiate them - are lacking ${ }^{1}$. Indeed, responses to outbreaks can be described as having a narrow therapeutic range where a small misstep can lead to catastrophic consequences for the affected populations. This phenomenon is especially pronounced when local health systems are weakened by inadequate infrastructure, underdeveloped processes, and critical shortages of skilled health professionals. In times of acute crisis, these system challenges result in devastating effects locally, which then affect the wider world indirectly through hysteria, isolationism, stigmatization, and economic 
destabilization. And with people traveling more frequently and over greater distances than in the past, health systems deficits can result directly in global spread of disease. Experts suggest that the battle against emerging infectious diseases will continue for some time, so it is important that we find a model to protect against future threats ${ }^{1}$.

Reflection on global planning for and responses to emerging infections provides important lessons of how to improve this therapeutic regimen. It is clear that failure to acknowledge disease severity, delay in response, and misaligned type of response leads to worsening of the epidemic. This combination initiates a doseresponse cycle that exists regardless of the infecting organism and is difficult to alter. A haunting recent example of this comes from the Ebola epidemic. Despite multiple outbreaks since 1976, response to the 2014 outbreak was delayed, claiming more than 1000 lives before WHO declared it an international crisis. With malaria, although a global eradication campaign was initiated in the mid 1990s, it was abandoned before the control infrastructure was strong, leading to a subsequent rise in morbidity and mortality. Despite decades of devastatingly high mortality from HIV/AIDS in Sub-Saharan Africa, it was not until 2004 when systems initiatives enabled developing countries to reliably access highly active antiretroviral therapy (HAART). And failure to sustain initial reductions in tuberculosis infection rates has led to more resistant disease today. The timelines, responses, and impacts for representative emerging and re-emerging epidemics in Sub-Saharan Africa can be seen in Figure 1. 
To treat these challenges locally and to vaccinate against future risk globally, some have called for responses that are better aligned with local needs, ie, a greater focus on surveillance, health systems strengthening, and training of health professionals from low resource settings ${ }^{2,3}$. Essentially, the goal is to employ strategies that maximize total exposure to therapeutic interventions over time in order to build local capacity, infrastructure, and systems to address emerging infections. This strategy can be interpreted much like the area under the curve (AUC) calculation is used for drug dosing and monitoring.

The prevailing model for international development is essentially bolus injections of reactive funding, external technical assistance, and uncoordinated training efforts as brokered by aid organizations such as the Global Fund, the World Bank, and USAID ${ }^{4}$. The effects of this treatment are rapid but the half-life is short; dissipating quickly once the bolus is discontinued. In contrast, a continuous infusion of a lower dose of the treatment is more likely to achieve steady state (stable healthcare systems) by allowing effects to build at a pace that is locally responsive ${ }^{4}$. In the short term, however, this regimen produces a lower peak concentration for addressing the crisis at hand. Combination dosing - including both loading and maintenance drips - leads to the greatest improvement in AUC by yielding not only the immediate response required to address emerging infectious diseases but also the capacity to sustain these gains in the face of future threats. Once the health system achieves steady state, we predict there will also be spillover gains for addressing other health challenges, similar to those seen with the transition of HIV/AIDS infrastructure to address additional chronic diseases beyond HIV/AIDS in 
Sub-Saharan Africa. Figure 2 illustrates how principles of pharmacokinetics/pharmacodynamics can be applied to health systems strengthening for emerging infectious diseases.

One national example of this combination dosing approach is the US partnership with the government of Rwanda to create a Human Resources for Health (HRH) model which will train thousands of new healthcare providers locally within seven years ${ }^{5}$. Of particular importance in Rwanda's ambitious plan is its focus on interprofessionalism as a way of highlighting the strengths of different fields in order to fill gaps in quality of care. Lessons learned from this initiative could also benefit the struggling US health system ${ }^{3}$. Parallel to this project is an expansion of and support for new cadres, such as community health workers, to address many healthcare needs without the lengthy periods of additional training associated with physician specialization. This is important because it emphasizes more creative, contextualized solutions that fit the phenotype of the local system rather than forcing strategies that have been designed for and tested only in settings with more resources. Continued monitoring of these projects is important, however. While key health indicators such as under-five mortality rates are improving, there can be unintended negative consequences of educational programs. For example, as health providers are trained, they may see greater opportunity in leaving the public sector to work in the urban private sector. Without appropriate human resources planning and incentives, this can result in a failure to meet the underlying healthcare needs of underserved populations. 
Note that this combination-dosing regimen need not be dependent on longterm donor support of financing or human resources. Rather, if outside agencies collaborate with local leadership to employ a systemic capacity building perspective - focusing not just on training of individuals but also on resourcing a healthcare infrastructure that rewards good performance, the treatment may exhibit a post antibiotic effect (PAE) such as that described with aminoglycosides. During this window, local efforts to create robust systems through scaling up and skilling up health providers from a variety of cadres must be prioritized. This will ensure that sufficient quantities of highly qualified staff will be able to work in systems that are resourced and incentivized to be successful while ensuring the population will be protected against future emerging threats.

Clinical pharmacologists analyze how medications are liberated, absorbed, distributed, metabolized, and excreted. They also estimate total drug exposure over time to minimize toxicities and maximize positive effects. These same principles predict the potential for combination approaches in addressing emerging infections through health systems strengthening. This enduring approach includes focused investment during crises in order to address the immediate need with a portion of that support set aside to build functionalities in the system in order to sustain the gains in the response to these diseases. 


\section{References}

1. Morens, D. M. \& Fauci, A. S. Emerging infectious diseases: threats to human health and global stability. PLoS Pathog.9, e1003467 (2013).

2. Gates, B. The next epidemic--lessons from Ebola. N. Engl. J. Med.372, 1381-1384 (2015).

3. Committee on Investing in Health Systems in Low- and Middle-Income Countries, Board on Global Health \& Institute of Medicine Investing in Global Health Systems: Sustaining Gains, Transforming Lives. (National Academies Press (US), Washington (DC), 2014).

4. Matowe, L. It's time to change the approach to capacity-building on supply chain management. Glob. Fund Obs. (2015).at <http://www.aidspan.org/gfo_article/itstime-change-approach-capacity-building-supply-chain-management> Accessed June 28, 2015

5. Binagwaho, A. et al. The human resources for health program in Rwanda--new partnership. N. Engl. J. Med.369, 2054-2059 (2013). 
Figure legends

Figure 1: Reactive responses to select emerging and reemerging epidemics

Figure 2: The kinetic/dynamic model of development assistance in response to emerging infectious disease epidemics 


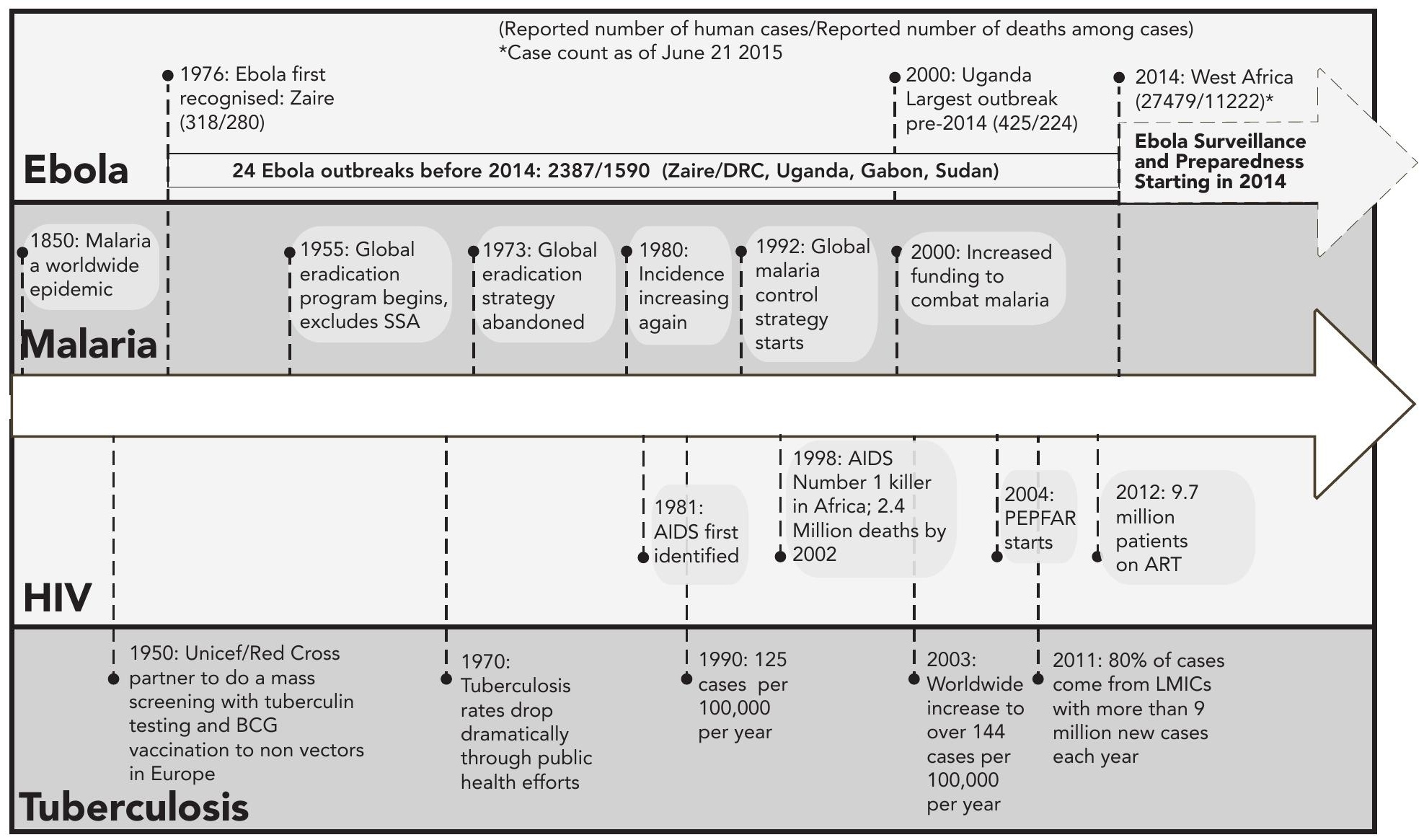




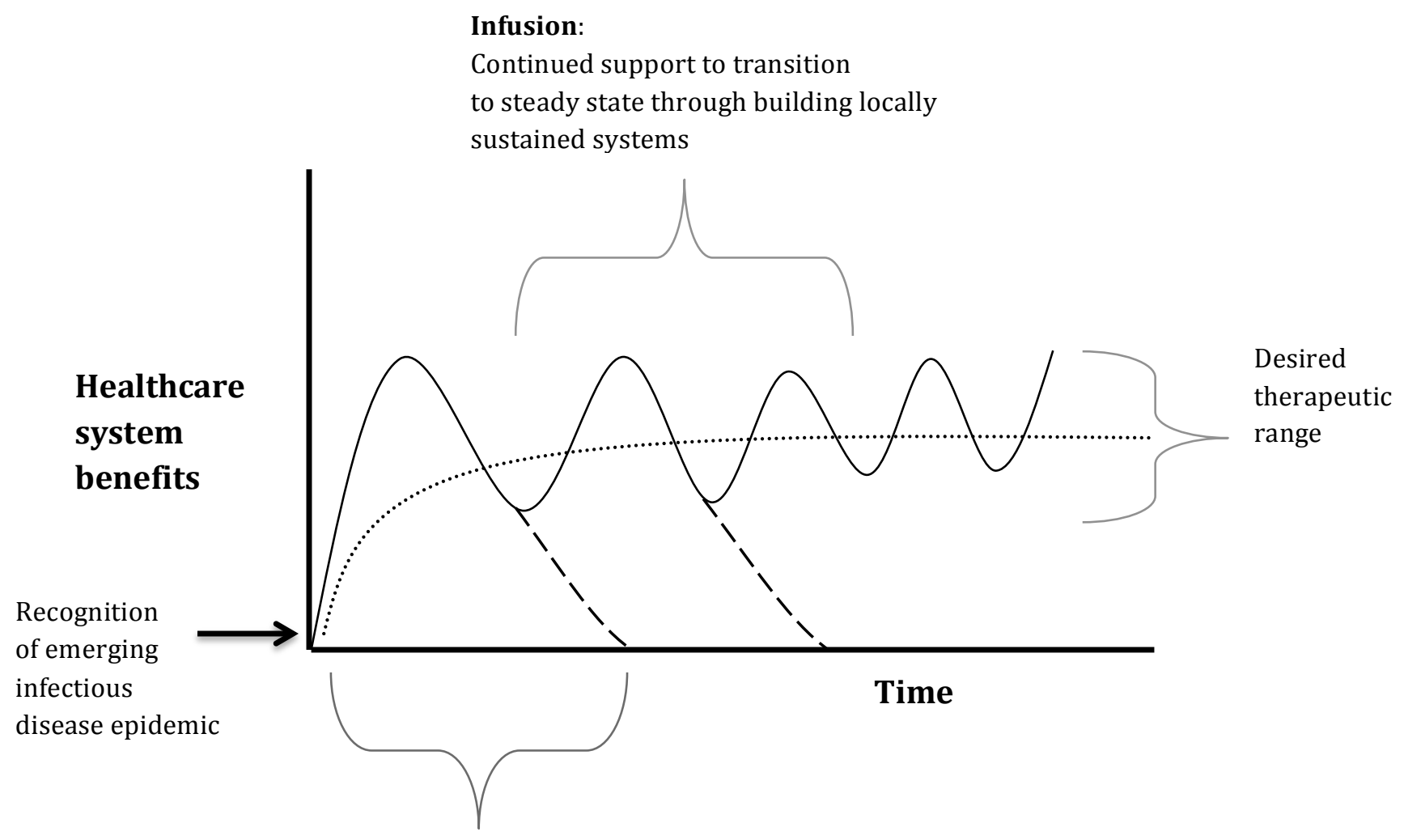

Bolus:

Initial injection of support to address urgent needs

Dashed line - Diminution;

premature withdrawal of

support leads to a rapid

decline in effect
Dotted line - Steady state;

sum of all parts is a locally

supported system responsive to current needs and future health threats despite withdrawal of outside support 